\title{
¿CÓMO ESTABLECER DE MANERA ADECUADA LOS HECHOS DE UNA TUTELA?
}

\section{HOW TO PROPERLY ESTABLISH THE FACTS OF AN ACTION FOR PROTECTION?}

\author{
WiLSON YeSid SuÁREZ MANRIQUE*
}

RESUMEN: El presente escrito parte de la insatisfacción de la manera en la que se ha abordado, por el ideario tradicional, la redacción de los hechos en una acción de tutela, en donde se ha obviado el poder narrativo, argumentativo, simbólico y trascendental que ostenta esta labor. Luego, pretende presentar, desde la retórica y la lingüística, la redacción de los hechos de la tutela como un proceso complejo, reflexivo, definido por diversas etapas, siempre provisional, que puede ser enriquecido por una constante deliberación, un diseño general de la acción y ciertas estratagemas que procuran hacer más eficiente esa tarea.

Palabras clave: Hechos, interpretación, lenguaje, estrategia, decisión.

ABSTRACT: This study stems from of the dissatisfaction of the way it has been addressed by the traditional view, the wording of the facts in an appeal for protection, where it has avoided the narrative, argumentative, symbolic and momentous power that holds this task. Then, it intends to present, from rhetoric and linguistics, that establishing, the facts of an appealfor protection is a complex process, reflective, defined through various stages, always provisional, which can be enriched by a constant deliberation, a general design of the action and certain schemes that seek to do the job more efficiently.

Keywords: Facts, interpretation, language, strategy, decision.

\section{INTRODUCCIÓN}

El éxito de una acción de tutela depende en gran parte de la forma como se establezca el acápite de los hechos ${ }^{1}$. La fortuna de ser beneficiado o la desventura de ser perjudicado por la decisión de un juez de tutela, dependen, en múltiples ocasiones, de la forma como el jurista interprete, diseñe y establezca los hechos de la acción de tutela. Pese a lo anterior, en el imaginario tradicional se sigue otorgando un peso superlativo a las consi-

* Doctor en Derecho de la Universidad, Colombia,de Salamanca. Profesor e investigador de la CURN, Dirección postal Calle 200 N 14-50, código postal 681004. Dirección electrónica: wilsonyesidsuarez@gmail.com

1 La tutela, en el ordenamiento jurídico colombiano, según el artículo 86 de la Constitución, es una acción pública, de carácter constitucional, instituida para la protección de los derechos fundamentales mediante un procedimiento preferente y sumario. Esta acción constitucional de protección de derechos es en esencia similar a las acciones de amparo, seguridad o protección, que se encuentran establecidas en la mayoría de las constituciones latinoamericanas. Por ejemplo, es análoga "al recurso de protección" establecido en el artículo 20 de la Constitución chilena, a la acción constitucional de amparo establecida en el artículo 103 y 107 de la Constitución mexicana, o a los procedimientos instituidos en el artículo 43 de la Constitución argentina, el 48 de la de Costa Rica, o el 88 de la ecuatoriana. Salvo las diferencias de nombre y algunas cuestiones terminológicas y técnicas, la finalidad de dichos procedimientos es similar; sus rasgos, presupuestos, características generales, y trámite sumario e informal, ostentan un parecido de familia. 
deraciones jurídicas, descuidando el poder simbólico, narrativo, y la influencia que puede ejercer la redacción de los hechos en la decisión el caso.

No se desea, con lo anterior, restar importancia a las consideraciones jurídicas y probatorias dentro del trámite de la acción o tutela, sino de resaltar un sector que pareciese olvidado en la práctica jurídica: el diseño y establecimiento de los hechos de dicha acción constitucional; esta parte del trabajo del jurista, por las características especiales de la acción de tutela ${ }^{2}$, se ha visto como algo con poca importancia, algo rudimental y técnico que no requiere mayor reflexión ${ }^{3}$. Se ha desconocido el lugar predominante que ocupa ${ }^{4}$. Sin embargo, no hay que perder de vista que los hechos también pueden ser interpretados, que las modificaciones a los estados de cosas, procesos y sucesos ${ }^{5}$, al ser traducidos al lenguaje son dotados de un nuevo significado, que se encarga a su vez de producir un efecto jurídico diferente dependiendo de la forma en que sean traducidos al derecho.

El presente escrito tiene por finalidad responder al cuestionamiento de su título, en el sentido de establecer una alternativa a la forma de narrar los supuestos fácticos y valorativos de la acción de tutela, y resaltar la importancia que esta labor tiene para la práctica jurídica. Para dar respuesta al cuestionamiento, y cumplir con los objetivos mediatos e inmediatos que se llevan de manera inmanente, se parte de una construcción expositiva, que

\footnotetext{
2 La acción de tutela es de carácter público, esto quiere decir que puede ser impetrada por cualquier persona (incluso personas jurídicas); no se requiere abogado para su presentación. La acción puede ser incoada ante cualquier juez, no existe una limitación constitucional para ello (existen algunas directrices administrativas consignadas por la Corte Constitucional en el auto 071 de 2001, sin que esto suponga una limitación de la competencia fundamental). La acción puede ser presentada de forma escrita u oral. Su trámite es preferente y sumario. El juez que conozca la acción cuenta con 10 días hábiles para resolverla. El juez que avoque el conocimiento de la tutela notificará de oficio o a solicitud de parte, en la forma más expedita, a las personas que puedan ser afectadas dentro del proceso. El juez tiene amplios poderes para el decreto y la práctica de pruebas y medidas cautelares. Sus sentencias consisten en órdenes de protección de derechos fundamentales. Las cuales pueden hacer uso de potestades ultra y extra petita. La decisión de la tutela puede apelarse, y eventualmente es revisada por la Corte Constitucional.
}

3 En este sentido, el Decreto 2591 de 1991, en su artículo 14, consagra el principio de informalidad, según el cual la acción podrá ser ejercida, sin ninguna formalidad. Para la Corte Constitucional, en la sentencia T 288 de 1997, la acción de tutela tiene un carácter informal que, por su misma naturaleza, riñe con toda exigencia sacramental que dificulte el sentido material de la protección que la Constitución quiere brindar a las personas por conducto de los jueces. Ese mismo carácter se refleja en un segundo principio, el de oficiosidad, que exige del juez de tutela, en ejercicio de su función constitucional, un papel activo, no solo en la interpretación de la solicitud de amparo -recuérdese que la persona que ejerce la acción no requiere ser experta en Derecho ni pulir extraordinariamente su lenguaje para acceder a la administración de justicia-, sino en la búsqueda de los elementos que le permitan comprender a cabalidad cuál es la situación que se somete a su estudio, para evaluarla a la luz del ordenamiento fundamental -que se presume conoce- y para adoptar una decisión justa que contemple la integridad de la problemática planteada y le dé solución adecuada con miras a proteger efectiva e inmediatamente los derechos afectados.

4 Gran parte de la astucia y la estrategia jurídica ha recaído en la interpretación de las normas jurídicas, de cierta forma el poder de interpretar o decir el derecho, ha ostentado una importancia superlativa en la solución de los conflictos jurídicos.

5 Hay tres tipos de hechos: estados de cosas, procesos y sucesos. "La superioridad en lo que respecta la población de un país sobre otro es un estado de cosas genérico, del que la actual superioridad, en lo que respecta a la población de Inglaterra sobre Francia es una ejemplificación”. "La lluvia es un proceso genérico, del que caer la lluvia en un determinado lugar y tiempo es una ejemplificación. Morir es un suceso genérico, del que, digamos, la muerte de César es una ejemplificación.” VON WRIGHT (1979) p. 47. 
en ocasiones se citará directamente y en otras se entenderá implícita; la cual se encuentra compuesta por los siguientes actores y factores: el abogado tradicional (AT) ${ }^{6}$, el abogado reflexivo (AR), quien sostendrá, de manera representativa, las principales tesis de este escrito, el titular de los derechos fundamentales (TF), el juez al que se le dirige la tutela (JM), la solución que se busca $(\mathrm{S})$, los posibles hechos que se desean establecer $(\mathrm{H})$, las variantes genéricas de los hechos pueden establecerse como (H1, H2...), y la forma de conectar los hechos con la solución mediante normas jurídicas $(\mathrm{NJ})$. De forma más específica puede decirse lo siguiente: un titular de derechos fundamentales, TF, acude a un abogado, AT o AR, para que instaure una acción de tutela en miras a conseguir que JM establezca $S$, en donde $\mathrm{H}$ es una variable que posee un peso considerable.

Este escrito está dirigido especialmente a los "prácticos" del derecho y a aquellos que inician su carrera como litigantes. Más allá de dictar un sistema erudito de estratagemas para tener razón ${ }^{7}$ o realizar un buen contrainterrogatorio ${ }^{8}$, pretende traslapar algunas sugerencias que desde los cantones epistemológicos citados podrían compartirse con los abogados que se enfrentan a la redacción de los hechos de una tutela.

Una aclaración preliminar que hay que tener en cuenta es que la forma de establecer los hechos varía dependiendo del tipo de tutela de la que se trate. Teniendo en cuenta la naturaleza de los derechos invocados y los modos en los que estos están presuntamente vulnerados, las estrategias a utilizar pueden ser muy distintas. Un análisis de este tipo sería muy pragmático y aportaría profundamente en el debate, sin embargo no es el objeto de este escrito. A esta situación podría llamársele teoría particular ${ }^{9}$ del establecimiento de los hechos de la tutela, y se aplicaría a casos como las sugerencias que deben efectuarse cuando se trata de proteger el derecho a la igualdad, la libertad o la vida. Sin embargo, una situación más general y abstracta, que puede aportar en un estadio más basto, podría ser llamada como: reflexión general del establecimiento de los hechos de la tutela; no obstante la eficacia de la teoría particular, su aporte es considerable en cuanto puede prestar mecanismos o situaciones de reflexión en la mayoría de acciones de tutela.

En Colombia la acción de tutela es la actuación judicial más usada para la protección de los derechos fundamentales y ha sido el punto de entronque de los derechos fundamentales con las prácticas jurídicas formalistas. Según DEJUSTICIA, en la encuesta nacional de necesidades jurídicas, realizada en el 2016 y publicada en el 2017, el 82\% de las personas que manifiestan conocer la acción de tutela, la han utilizado para solucionar un conflicto. La tutela ha supuesto el cambio de la forma como se piensa y evoluciona el Derecho en el ordenamiento jurídico colombiano, especialmente, por cuanto ha permitido su materialización y el diálogo con los derechos fundamentales. El hecho de ser una acción pública, que puede ser instaurada por cualquier persona, informal, con requisitos poco rigurosos,

\footnotetext{
$6 \quad$ La noción representa por AT es un ideario en el que se encuentran las percepciones generales sobre la práctica jurídica, especialmente, las que son dominantes en el campo de la acción de tutela.

Schopenhauer (1997) p. 7.

Carofiglio (2010) p. 25.

Alexy (2002) p. 35.
} 
que supone amplios poderes de dirección judicial, la han convertido en un punto central de debate jurídico y un asunto de interés público.

Este escrito se divide en tres partes. En la primera se establecen los presupuestos generales de los que parte la reflexión que se presenta; presupuestos que deben ser aceptados para entender el sentido del artículo; se defienden y explicitan cuatro ideas generales derivadas de la multiplicidad y complejidad del universo fáctico y jurídico. En la segunda parte se exponen tres nociones generales: el proceso de tutela como una contraposición de argumentos, el entendimiento de los hechos de la tutela como argumentos, y la posibilidad de aplicar los criterios de racionalidad judicial en el establecimiento de los hechos de la acción. En la tercera parte, que se considera el núcleo central del trabajo, se realiza, teniendo en cuenta lo anterior, la reconstrucción de tres líneas expositivas: dos puntos de partida, la redacción como un proceso de distintas etapas siempre provisionales, y la explicación de algunas estratagemas referidas a los hechos.

\section{PRESUPUESTOS GENERALES Y LAS ELECCIONES BÁSICAS}

El mundo social está constituido por un entramado de hechos, estados de cosas, procesos y sucesos que influyen de manera notable en la interpretación de las personas ${ }^{10}$; a su vez que son el reflejo de esa interpretación. Es muy común que parte importante de los documentos jurídicos, de distinta índole, estén compuestos por un acápite de hechos, en sentido genérico ${ }^{11}$. Cuando se trata de establecer en una demanda o en una acción cualquiera determinado hecho se pretende especialmente justificar una norma jurídica en sentido amplio, o solicitar que se aplique determinada consecuencia jurídica ${ }^{12}$. Se desea señalar que esos hechos que se han establecido son buenas razones para la aplicación de determinada norma jurídica; en sentido reducido esta idea se representa en la subsunción ${ }^{13}$.

Los cuatro presupuestos generales para que la problemática planteada tenga un sentido y pueda tener relativa utilidad son los siguientes: el mundo de los hechos que pueden ser relevantes para el derecho en el ámbito de la acción de tutela es muy amplio; al momento de presentar una acción de tutela el jurista escoge entre los diversos hechos que la realidad le presenta; esta escogencia se ve plasmada en la traducción que a palabras efectúa el jurista; y la escogencia de la forma como se narran los hechos tiene consecuencias jurídicas distintas o, por lo menos, influencian al juez en diversos grados.

Cuando se impetra una acción de tutela o de amparo, el jurista se ve enfrentado a una indeterminada cantidad de hechos que pueden ser, en sentido genérico, considerados como jurídicos, especialmente, en el campo constitucional ${ }^{14}$; esto dista de las concepciones

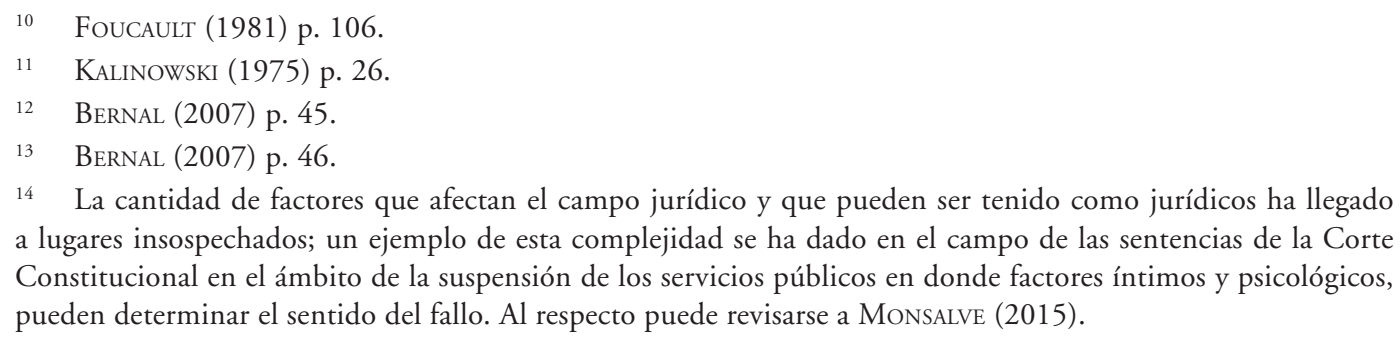


tradicionales que el legalismo y la concepción del ejercicio del derecho como una simple subsunción ha estandarizado ${ }^{15}$. Por la ambigüedad estratégica de los derechos fundamentales y los principios, la concepción ampliada del supuesto de hecho de los derechos fundamentales ${ }^{16}$, y la vacilante jurisprudencia ${ }^{17}$, se transforman y amplían la cantidad de elementos que pueden ser considerados como relevantes para el derecho en un caso específico. Situación que conlleva de una manera importante a complejizar la labor de extraer dentro de las situaciones factuales cuáles son las más relevantes para la protección en sede de tutela, o cuáles existen de manera jurídica, y, consecuencialmente, cuál es su efecto.

Luego, de esa cantidad de hechos, estados de cosas y procesos, el jurista debe escoger cuáles considera importantes para el establecimiento de su acción. No tiene sentido pretender que en una acción constitucional se narren de manera detalladas todos los hechos que giraron alrededor del caso; esto sería un trabajo abyecto y fútil. JR debe de manera adecuada reducir la complejidad, de conformidad con el diseño de su plan de tutela, escogiendo dentro del universo posible de los hechos jurídicos de su tutela, cuáles son los que le permitirán cumplir de mejor forma su cometido. Realiza, de manera indirecta, y no exclusiva, una primera consideración de los aspectos que fundamentan la protección de los derechos fundamentales. La escogencia de estos hechos luego será enriquecida y tensionada con la escogencia de las normas jurídicas y las pretensiones de la tutela ${ }^{18}$.

Los hechos escogidos son establecidos, en la mayoría de los casos, en un documento ${ }^{19}$, en el que se traducen, o por mejor decir, se plasman para que sean revisados posteriormente por el juez, a efectos de estudiar, corroborar y decidir. El establecimiento de los hechos en el documento refleja una escogencia en dos sentidos; en primer lugar, la elección dentro de las múltiples posibilidades de las situaciones fácticas que considera con mayor relevancia jurídica para soportar sus pretensiones; en segundo lugar, muestra la escogencia de diferentes palabras con los cuales se traducen esos hechos al lenguaje jurídico, una situación factual puede ser representada en tantas formas posibles como competencias lingüísticas ostente el jurista. Las palabras vienen a representar ideas, por lo que los hechos pueden ser representados con distintas palabras ${ }^{20} \mathrm{y}$ formaciones semánticas y sintácticas.

El efecto persuasivo o heurístico ${ }^{21}$ de las palabras en el juez y en la solución de caso, significa que dependiendo las palabras que se establezcan dentro de la tutela, las formas semánticas a las que se traduzca, el sentido del fallo puede variar. La fuerza del hecho dentro de la argumentación resulta ser una verdad evidente para el litigante; considerar lo contrario sería una gran ingenuidad. El éxito de una acción de tutela radica en un grado impor-

\footnotetext{
15 BERNAL (2007) p. 45.

16 LOPERA (2010) p. 48.

17 SuÁrez (2015) p. 106.

18 La tutela ha de entenderse como una totalidad, en la que los hechos, las pretensiones, las pruebas y los fundamentos jurídicos, son las partes integrativas.

19 El artículo 86 de la CP, no requiere formalidad para la presentación de la acción de tutela. Y, el Decreto 2591 de 1991, en su artículo 14, deja abierta la posibilidad de que la acción de tutela se presente de manera verbal.

20 Fregue (1973), p. 56.

21 Hernández (2010) p. 48.
} 
tante en cómo se establezcan los hechos dentro de la misma, y la relación que se establezca con las demás partes del documento.

Lo anterior, pretende, en parte, cuestionar la concepción cronológica y simplista en la cual se ha restado gran importancia al establecimiento de los hechos en la tutela, y donde se considera que el quid del asunto radica en el conocimiento que el abogado tenga de los últimos movimientos judiciales sobre el tema; el principal debate es normativamente reducido. Esto no pretende caer en la inocencia de creer que el debate jurídico no es importante, ni desconocer cómo una dogmática de los derechos fundamentales ${ }^{22}$ delimita y condiciona el establecimiento de los hechos. Tampoco puede descartarse de plano cómo la complejidad fáctica limita el discurso puramente normativo; pues no es recomendable ver simplemente una influencia unidireccional.

\section{LOS HECHOS COMO PARTE DEL DISCURSO ARGUMENTATIVO}

En este apartado se desean explicitar tres ideas relacionadas: la importancia de entender la acción de tutela como un discurso argumentativo (una especie dentro del género de los procesos interpretativos constitucionales); $\mathrm{H}$ como parte o como una forma de argumentos dentro de la acción de tutela; y la importancia que se sigan los criterios de racionalidad en la argumentación jurídica en el momento de establecer $\mathrm{H}$ (lo que puede denotarse como un primer llamado a la reflexión del asunto).

"La teoría de la argumentación rechaza las antítesis demasiado netas; muestra que, entre la verdad absoluta de los dogmáticos y la no verdad de los escépticos, hay lugar para verdades susceptibles de ser sometidas a permanente revisión”23. En los procesos judiciales prima más la argumentación que la demostración. "En rigor, las verdades que produce el proceso son verdades históricas y no científicas ni formales" ${ }^{24}$. La interpretación constitucional se asemeja a un discurso argumentativo, en el cual a un grupo de argumentos se le contrapone otro grupo de argumentos, debiendo prevalecer al final los mejores argumen$\operatorname{tos}^{25}$. De lo que, en parte se sigue, que pueda entenderse para el caso, la acción de tutela como un campo de contienda de argumentos. Apartándose ello, del entendimiento de la acción como un área de demostración.

Así las cosas, la acción de tutela puede ser vista como un proceso constitucional, que se asemeja a un discurso argumentativo, en el que se establece un debate de argumentos, entre el accionante y accionado, que el juez se encargará de resolver, protegiendo o no los derechos fundamentales presuntamente vulnerados. Esta forma de concebir la acción de tutela implica la necesidad de fortalecer el diseño de los argumentos de la acción, en el cual se debe buscar la mejor forma de presentación de los mismos, su mayor peso y grado de eficacia. Dentro de los diferentes argumentos que pueden aducirse en este discurso argumentativo, se encuentra $\mathrm{H}$.

\footnotetext{
$22 \quad \operatorname{AleXy}(2002)$ p. 34.

23 Carofiglio (2010) p. 11.

24 Carofiglio (2010) p. 154.

25 Alexy (2000) p. 6.
} 
Los hechos de la tutela son argumentos dentro del proceso. Argumentos con un peso considerable, los cuales pueden ser establecidos de manera estratégica, y en este sentido es aconsejable que cumplan criterios de racionalidad similares a los exigidos a las decisiones de los jueces. La corrección de la decisión de los jueces de tutela, depende en gran medida de su adecuada justificación y racionalidad. "De esta manera, los criterios de racionalidad determinan el fundamento de las interpretaciones correctas, es decir, son criterios para la fundamentación razonable de las decisiones" ${ }^{26}$. La racionalidad se establece como sucedáneo o sustituto de la objetividad. Luego, los criterios exigidos para la racionalidad de las decisiones judiciales son un candidato plausible para iluminar la construcción y el establecimiento de los hechos en la acción de tutela.

Para Carlos Bernal, citando a Atienza, los criterios de racionalidad judicial son: i) la claridad y consistencia conceptual, ii) la consistencia normativa, iii) la completitud o saturación de la argumentación, iv) el respeto por las reglas de la lógica, v) el cumplimiento de las cargas argumentativas establecidas por la práctica constitucional, y vi) la consistencia y coherencia argumentativas $^{27}$. Estos criterios, como se dijo, alumbran la forma de redacción de los hechos de tutela, y demás consideraciones jurídicas, pueden ser entendidos como criterios de racionalidad de argumentación que pueden ser aplicados a la construcción de los hechos de la tutela.

Luego, una argumentación-hecho de la tutela se considera más racional en cuanto esté provista de claridad y consistencia conceptual y lingüística, que además pueda fundamentar los mismos resultados interpretativos cuando se aplique a los mismos supuestos, sea completa, es decir, contenga todas las premisas que le pertenecen, respete la lógica deductiva, y las cargas de la argumentación, carezca de contradicciones internas, y esté respaldada por reglas y principios generales ${ }^{28}$. Sin embargo, estas directrices no son el aspecto central de este escrito. Como sí lo son los criterios siguientes, en donde en un grado menos de abstracción se consideran algunas de estas reglas.

\section{HECHOS Y ESTRATAGEMAS}

El establecimiento de estratagemas sobre cómo deben redactarse los hechos de una tutela, teniendo en cuenta la ardua complejidad que existe en los $\mathrm{H}$ jurídicamente relevantes, su necesaria escogencia, su traducción, su efecto, y su poder argumentativo dentro de un proceso interpretativamente adversarial en el que se busca que JM llegue a $S$, puede ser un centro interesante de reflexión.

AT entiende este proceso de escogencia de $\mathrm{H}$ como una labor artesanal y rudimentaria que no conlleva gran deliberación. Su formación como abogado no estuvo enmarcada en amplias reflexiones sobre la fuerza argumentativa del lenguaje, su capacidad de persuasión, alcances y la capacidad de trasmitir emociones y sentimientos. Él busca, al mejor estilo clásico, una subsunción en un supuesto de hecho. Se aprovecha de que es una acción

\footnotetext{
26 BERnAL (2015) p. 32.

27 Bernal (2005) p. 27.

28 Bernal (2008) p. 211.
} 
informal, que puede redactar rápidamente, no la considera un proceso continuo y provisional, y no presta gran atención a las palabras ni su efecto ${ }^{29}$.

Por su parte $A R$, en su permanente ejercicio reflexivo ${ }^{30}$, busca algunos puntos de partida y algunas reglas básicas, intuitivas o producto de referentes teóricos mediatos, a efectos de enriquecer la construcción de la forma más adecuada de narrativa fáctica en busca de $S$ mediante H. Para ello instituye dos puntos de partida generales que sirven de marco para materializar la reflexión y cierto tipo de reglas a tener en cuenta en la redacción de $\mathrm{H}$ en aras de lograr una mejor influencia en la $S$ que otorgue JM. En ocasiones gran cantidad de tutelas pueden recibir una decisión negativa debido a la falta de pericia y reflexividad en el momento de redactar sus hechos. Los puntos de partida llevan a entender la redacción de los hechos como un proceso provisional y dinámico, que debe estar delimitado por ciertas estratagemas extraídas de la praxis jurídica y la crítica racional.

Existen dos puntos de partida para la redacción adecuada de los hechos en una tutela: el primero, refiere a entender $\mathrm{H}$ como una parte fundamental de la tutela que obedece a un diseño estratégico; $y$, el segundo, a que este diseño debe estar precedido de un estudio ajuiciado del caso.

El primer punto conlleva a que AR comprenda el poder argumentativo, persuasivo y la capacidad de transformación que contiene la forma como redacte H. Esto le implica la necesidad y el deseo de adentrarse en las profundidades de la complejidad fáctica y jurídica respectiva, de esforzarse por tener la visión más completa del asunto que está proyectado. $\mathrm{Y}$, en contrapartida, le conllevará a estar más alerta, a ser más cuidadoso en el establecimiento de $\mathrm{H}$ en la búsqueda de $\mathrm{S}$.

En cuanto al segundo punto de partida, se consideran emblemáticas y representativas dos líneas argumentativas unidas por la misma idea. Genaro Carrio, en un sentido similar estableció: "el caso debe ser estudiado bien, lo mejor posible". Para él "Un abogado que sabe derecho y que estudia bien los asuntos que le toca intervenir lleva enormes ventajas al de su colega que lo maneja atientas" ${ }^{\prime 1}$. De que los jueces no estudien bien los casos no se sigue que es malo que los abogados los estudien bien. En un sentido similar Carofiglio estableció como su principal estratagema: "La primera condición para interrogar bien es prepararse bien" 32 .

Así pues, la comprensión del poder persuasivo, argumentativo y transformador de $\mathrm{H}$ y la exigencia de un estudio y una preparación adecuada, si bien es cierto no garantizan el éxito de obtener $S$, si presentan ventajas comparativas respecto de quien no obre teniendo esos lineamientos en mente. AR gana mucho, si bien no tiene seguro nada.

La redacción adecuada de $\mathrm{H}$ es un proceso, como todo proceso está compuesto de múltiples etapas. Esta visión de un proceso se opone a una consideración de la estructuración de $\mathrm{H}$ como un asunto de terminación precaria, artesanal y rudimentaria donde la experticia jurídica y las competencias del abogado no son muy importantes. Para efectos

\footnotetext{
29 Austin (1990).

30 Alexy (2008) p. 25.

31 Carrio (1995) p. 16.

32 Carofiglio (2010) p. 25.
} 
expositivos se consideran importantes por lo menos tres etapas ${ }^{33}$, al momento de la redacción de $\mathrm{H}$ : la preparativa, la de diseño, y la de revisión.

En el primer caso lo que el jurista debe hacer es obtener la mayor cantidad de información fáctica y jurídica sobre el asunto del cual trata. No puede realizarse una buena interpretación fáctica de los hechos si a la par no se realiza una muy buena revisión y adecuación jurídica (sin embargo en este escrito solo se ocupará del primero de los eventos). AR debe partir de que los hechos relatados por TF solo constituyen una interpretación subjetiva de cómo sucedieron las cosas, interpretación que aunque importante puede ser mejor diseñada teniendo en cuenta la finalidad de la acción y el plan que se haya realizado.

Debe solicitar a TF que enmarque por escrito con el mayor de los detalles posibles todas las situaciones fácticas importantes y sus pretensiones ${ }^{34}$, a efectos de tratar de hacerse una idea global de lo que ha sucedido, lo que desea TF y permitir una mejor actuación. Si es posible entrevistarse con testigos o demás personas que conocieron de la situación, sería conveniente. Nunca debe pasar por alto la posibilidad de que TF haya mentido, u ocultado o alterado información (debido a cuestiones íntimas, personales, de buen nombre, prejuicios, etc.).

Luego de obtener mayor conocimiento del caso, el jurista debe formarse una opinión independiente de la narrativa de $\mathrm{H}$ y su relación con NJ y S. Sin importar que la nueva narrativa, en principio imparcial, pueda ser diferente a la que reconstruyó en un primer momento TF, y, sin perjuicio, que sea solo una historia provisional ${ }^{35}$.

Alguien podría pensar que esta situación en muchos casos es innecesaria, que constituye una actividad muy costosa que se puede obviar y en últimas que se puede suplir con una tarea diligente del juez (que en último caso deberá realizarlo al momento de fallar). Sin embargo, quien conozca de mejor forma el caso lleva ventajas respecto del jurista que quiera resolverlo con poca preparación. El hecho de conocer de la mejor forma el asunto presenta ventajas comparativas que se materializan en la práctica y que permiten un mejor diseño y condicionamiento con los argumentos jurídicos. Podría establecerse como hipótesis tentativa que los costos que se invierten ex ante de presentar la tutela son costos que se ahorran con la solución del caso o en la estrategia posterior a esta; en estos casos los costos ex ante son costos que reducen los costos ex post ${ }^{36}$.

Luego de obtener el mejor acercamiento posible a las situaciones fácticas que se encuentran implicadas en el caso, se debe realizar la segunda etapa del proceso, la cual está compuesta por dos eventos: una redacción provisional de los hechos de la tutela, la cual debe ser obra como hipótesis de trabajo ${ }^{37}$, y, luego contrastando los aspectos provisionales con el "test de redacción", compuesto con los estratagemas que se explicitan más adelante, a efectos de determinar si se cumplen las sugerencias mínimas de claridad, simplicidad, orden, forma y dirección de redacción.

\footnotetext{
DWORKIN (1992) p. 57.

CARrio (1995) p. 16.

Carrio (1995) p. 16.

Calderón (2010) p. 148.

Pizarro (1997) p. 33.
} 
La tercera etapa se realiza una vez se tenga un primer proyecto de tutela. Su objeto principal es hacer más coherente los hechos con las pretensiones, pruebas y fundamentos jurídicos, lo que puede ser llamado coherencia interna de la tutela, por una parte, y revisar la coherencia externa de la tutela por la otra, es decir, su relación con los deseos de TF y las posibles consecuencias indirectas que pueda $\operatorname{traer}^{38}$.

Además de ello, en esta etapa de revisión, AR debería, luego de perfeccionar su intuición de la redacción primaria de los hechos con el test, dejar reposar el escrito y no entregarlo hasta tanto no haya trascurrido un tiempo prudencial. Debe alejarse del documento por unas horas o días para permitirse una nueva visión. En este tiempo puede pedir un consejo o una visión de un tercero acerca de la manera como se establecieron los hechos. Alguien ajeno al caso, puede tener una impresión no contaminada, similar a la que puede dársele a JM.

A continuación se representan doce estratagemas para la redacción de los hechos de la tutela. Primera: se deben redactar los hechos de la forma más clara y simple posible. AR debe ser un facilitador del proceso de comunicación con JM, quien es el receptor (en el mejor de los casos) de la información que establece. Hay que tener en cuenta que los hechos no se redactan para sí, sino para que otra persona, con limitaciones de tiempo y comunicación, y con múltiples problemas jurídicos a resolver, se encargue de emitir una $S$. Luego, presenta una gran ventaja estratégica la claridad en la información que se transmite. La claridad se opone a la ambivalencia, la confusión, y en general, a la oscuridad e indeterminación en la redacción ${ }^{39}$.

La simplicidad en la redacción no implica que se dejen por fuera aspectos relevantes al momento de la presentación de $\mathrm{H}$, sino que los hechos se establezcan de la forma más elemental posible. El establecimiento de los hechos de la manera más elemental posible, dentro de las estructuras básicas de sintagma nominal y verbal ${ }^{40}$, o bajo la clásica noción de sujeto, verbo y predicado, además de dotar de claridad e inteligibilidad al escrito, muestra orden, preparación y el conocimiento que el abogado ostenta del litigio ${ }^{41}$.

Segunda: no siempre el establecimiento de los hechos en un orden cronológico es la forma más adecuada de presentar $\mathrm{H}$. El establecimiento cronológico de $\mathrm{H}$, en donde la afectación de TF se denota en los últimos hechos de la tutela, puede presentar confusión debido a que en muchos casos no representa la afectación adecuada de los derechos fundamentales y puede distraer a la persona que recibe la información, sobre todo en aquellos casos en los cuales la naturaleza fáctica abunda. Por estas dos razones, se sugiere que en los primeros hechos se establezca la afectación de TF, para que con esta idea clara se permita a la JM el mejor entendimiento de $\mathrm{H}$. Es decir, que cuando en los primeros hechos se establece la afectación de una posición jurídica fundamental, los demás son entendidos con mejor claridad por el auditorio, pues, se le enmarca una finalidad que dota de mayor cohe-

\footnotetext{
38 Carrio (1995) p. 17.

39 Robles (1984) p. 84.

40 Gutiérrez (1997) p. 16.

41 Carrio (1995) p. 25.
} 
rencia al establecimiento de los demás supuestos factuales. Además de ello, la atención de JM se capta de mejor forma.

No existe restricción constitucional o legal que implique que los hechos deban establecerse de manera cronológica, de la forma en que se sucedieron en el tiempo. El poder establecer un orden diferente cuando se están redactando, puede ser una herramienta muy útil a la hora de presentar la acción. La sugerencia general es que se estudie, y se tenga como una alternativa plausible, la posibilidad de iniciar $\mathrm{H}$ con los supuestos fácticos más representativos que dan lugar a la vulneración de los derechos fundamentales así estos se hayan producido ulteriormente.

Tercera: la acción de tutela, por lo general, y especialmente en el caso de personas naturales, debe estar redactada en primera persona ${ }^{42}$. Si se establecen de forma impersonal, como cuando TF es un sujeto de una historia, quizás diferente al del proceso, se tiene menos peso argumentativo a cuando se redacta la tutela en primera persona. Esto se debe principalmente a tres circunstancias: el titular se encuentra involucrado en el proceso, tiene más certeza de la forma como ocurrieron los hechos, y el juez se tendrá que dirigir a él directamente en la resolución del caso.

La presentación de $\mathrm{H}$ es un diálogo íntimo entre TF y JM. El establecimiento de intermediarios puede implicar lo impersonal de una acción tan personal y que se encuentra ligada a las posiciones básicas del individuo en sociedad. Si se redacta en primera persona existirá más cercanía entre la comunicación, lo cual permitirá un mejor flujo de la información, y revelará un interés más directo del accionante. A su vez, la redacción personal de los hechos de tutela puede verse indirectamente como una forma más precisa de mostrar cómo sucedieron los hechos desde la perspectiva de quien se ve afectado por la presunta vulneración, lo cual tiene mayor peso argumentativo a cuando alguien que no estaba involucrado en los pormenores del evento los establezca y redacta como si los hubiera vivido, situación que puede restar credibilidad a la narrativa que establezca. Lo cual, además de involucrar a la víctima dentro del proceso, hará que las medidas que tome el juez tengan que dirigírsele directamente a TF y no a un tercero, lo que de manera inconsciente permite una mayor carga en la conciencia del juez a su vez que reta la empatía ${ }^{43}$ para con la protección de los derechos fundamentales.

Cuando se sugiere que se redacte en primera persona no quiere decirse que sea el titular quien establezca los hechos de la tutela, esta labor corresponde a AR, en virtud del diseño del plan de la acción. Quiere decirse que, teniendo en cuenta los beneficios que ello trae, sea el titular del derecho quien suscriba la acción y sea el emisor de $\mathrm{H}$.

Cuarta: AR debe comprender que las situaciones fácticas pueden ser traducidas al lenguaje de múltiples maneras, y debe elegir la que resulte más convincente y conveniente. El poder del lenguaje debe llevar a que el abogado transmita con éxito el mensaje. En la acción de tutela se necesitan competencias lingüísticas desarrolladas para escoger cuidadosamente las palabras que van a ser utilizadas, sobre todo debido a la carga emocional que ellas implican y las existencias de hechos jurídicamente valorativos que pueden ser esenciales

42 LYONS (1997) p. 14.

43 Lo Cascio (1998) p. 48. 
para decidir el asunto ${ }^{44}$. Una redacción fría de los hechos no da lugar a transmitir la afectación de los derechos fundamentales. Una frase del tipo "se trasgredieron los protocolos de seguridad en la cárcel” es distinta a una frase en la que se manifiesta "se desnudó al recluso, obligado a agacharse o hacer flexiones de piernas y a mostrar sus partes íntimas a la guardia”. El ejemplo es muy extremo, pero resulta necesario establecerlo de esta forma para que sea más comprensible.

Dentro de los diferentes actores importantes del derecho existe una delimitación un poco clara de los roles y funciones que se presentan como canónicos y que son atribuidos a determinadas personas. Así pues, la función, el rol y el producto, del legislador, el jurista y el juez son diferentes ${ }^{45}$ (esto puede enriquecerse con el discurso dirigido a la academia, los estudiantes, los ciudadanos, etc.). Teniendo esto en mente se sugiere la siguiente estratagema.

Quinta: Se debe partir del hecho que la acción de tutela es, en una de sus vertientes, un hecho de comunicación en el cual TF se dirige a AJ, su auditorio ${ }^{46}$, para que resuelva el caso o tome una decisión $S$, por lo que el accionante debe tener claro que los hechos no los escribe para sí mismo, ni son hechos dirigidos al legislador, ni a unos estudiantes, sino que $\mathrm{H}$ se dirige a JM. Luego la forma como se establezcan debe hacer parte del diseño no de una política pública no de una hipótesis académica, sino de una narrativa que tiene por finalidad que se tome $\mathrm{S}$, basado en $\mathrm{H}$ y $\mathrm{NJ}$, en un caso particular. Esta forma de entender la dirección que deben tomar los hechos de la tutela se fundamenta fácilmente en concepciones acerca del auditorio en la argumentación y el entendimiento que JM, es, la mayoría de las veces, en primera instancia, un juez municipal o del circuito con múltiples asuntos pendientes.

Sexta: JR debe partir de la falta de tiempo que tiene el juez para tomar $S$, de la gran carga que existe en los despachos, la falta de personal y técnica en la resolución de los litigios, y en virtud de ello, establecer el menor número de hechos en la tutela. Se debe distraer de la menor manera la atención de la persona encargada de llegar a S, para así evitar distorsiones innecesarias. El principio de necesidad rige dicho establecimiento.

Séptima: JR debe tratar de que con $\mathrm{H}$ se genere la mayor empatía ${ }^{47}$ posible con JM. Es decir, de que al transmitírsele con las palabras adecuadas la vulneración o amenaza a los derechos fundamentales él, además de entender que se trata de una situación grave en la que hace falta su intervención, considere que es una situación en la que se puede encontrar inmerso él como persona o sus familiares. Con ello se ganará que él tienda a tomar la decisión que él cree se debería tomar en caso de que el perjudicado o amenazado en esa situación concreta es él; que la decisión que tome el juez es la que debería tomar si él fuera quien instaurara la acción de tutela.

Octava: pese a que es importante que $\mathrm{H}$ sea lo más simple y claro posible, hay un tópico en el cual se hace necesario reparar en detalles, y es en el de la descripción vívida y

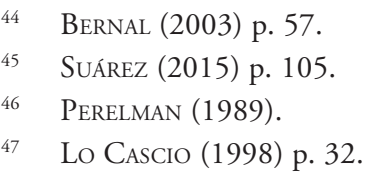


cruda, de cómo se afectan o amenazan los derechos fundamentales. La magia del lenguaje permite que en determinados asuntos y echando mano de recursos literarios, se busque graficar de la manera más realista y detallada de las afectaciones o amenazas de los derechos fundamentales. Para esto es necesario que el jurista se acerque a la literatura ${ }^{48}$, la cual, puede ayudar a generar el desarrollo de competencias que permitan mostrar de la mejor forma los sentimientos, sufrimientos, padecimientos y frustraciones que le acaecen a TF.

Novena: JR debe establecer patrones factuales de éxito y fracaso. Es importante que busque casos similares ya fallados, y se estudien, al igual que los que se fallaron a favor, los que se decidieron en contra. De los casos que fueron fallados a favor, puede extraer patrones recurrentes de los casos que han sido decididos positivamente. Luego, entre mayor se conozca el asunto mejores herramientas se tendrán para buscar una estrategia más eficiente. JR debe reflexionar en cada instante cuáles son las posibles situaciones factuales y jurídicas que alegará la contraparte para oponerse a la protección del derecho fundamental alegado.

Décima: La redacción de los hechos debe ser única, en el sentido de que cautive al juez; es importante llamar la atención sobre el caso que se presenta. JM se encuentra horadado de un número considerable de acciones de tutela, que parecen hechas en un formato frío, rústico y desinteresado. Si se desea que la tutela invite a reflexionar, y que se pase por alto la insana costumbre de declararla improcedente, se hace necesario que quien la lea pueda vislumbrar en ella rasgos particulares acerca del asunto, se hace necesario, mediante el lenguaje y la escogencia adecuada de los hechos, captar la atención de JM.

Undécima: el lenguaje utilizado en la redacción de los hechos debe ser muy cortés y prudente ${ }^{49}$. Si se utiliza lenguaje inadecuado en la acción, que por ejemplo sea vulgar, grotesco, y ofensivo respecto de la otra parte o se efectúan afirmaciones que no puedan ser soportadas, se llega a generar una imagen inadecuada de TF y AR. Una tutela redactada adecuadamente, con un lenguaje eficiente, cortés y elegante, genera una impresión positiva del accionante en JM. Muchas veces en los documentos jurídicos se dicen cosas más importantes con lo que se calla que con lo que se avienta. El sentido de la información que se está deseando transmitir no es solamente un acto explícito. Es importante darle la impresión al juez (aunque es más importante serlo), de que está tratando con una persona recta, respetuosa de la ley y que actúa de buena fe. Cuando los hechos de una tutela se cargan de expresiones agresivas, grotescas e hirientes contra la contraparte, en la mayoría de las ocasiones, más que descalificar a la otra parte, descalifican al emisor. Por eso hay que entender la imparcialidad estratégica y neutralidad de JM, como una situación que puede ser aprovechada. Algo similar a lo anterior, puede ser entendido bajo la concepción de máximos locales y máximos globales ${ }^{50}$; la satisfacción de nuestras necesidades no solo puede ser realizada de manera inmediata sino para el caso, mediata; teniendo en cuenta que es más importante la imagen ulterior que tenga el juez en el momento de fallar la decisión, que la inmediata que pueda resultar de la lectura de la tutela.

\footnotetext{
$48 \quad$ Nussbaum (1997) p. 85.

49 Carofiglio (2010) p. 24.

50 ELSTER (1989).
} 
Duodécimo: La redacción de los hechos debe tratar de plasmarse de la manera más cercana posible a los supuestos de hecho de las reglas o subreglas creadas jurisprudencialmente o actualizadas por el legislador. Se trata de un procedimiento que quiere llegar a facilitar la necesaria subsunción que en la mayoría de los casos debe realizarse luego de que el debate jurídico permite establecer la premisa mayor para la decisión del caso ${ }^{51}$. En otras palabras, para facilitar el éxito de la acción, deben poder adscribirse fácilmente en el supuesto de hecho amplio, que actualmente existe en el ordenamiento jurídico colombiano. Por tal razón, se debe ser muy cuidadoso en el entendido de buscar palabras que signifiquen o referencien en el sentido más cercano los adjetivos, sustantivos, verbos o adverbios que hayan sido establecidos como jurídicamente válidos en las reglas constitucionales en donde se condensan los derechos fundamentales que se desean proteger. En síntesis, un abogado reflexivo, aborda con seriedad y esfuerzo la redacción de los hechos de la tutela, conoce su potencial, está preparado y es proactivo. Siempre es generoso con buenas dosis de creatividad y originalidad.

\section{CONCLUSIONES}

La redacción de los hechos de la tutela dista de ser una actividad simple, en la que el poder reflexivo está ausente. La elección de los hechos jurídicos, su traducción a palabras, lo que significan estas, singular y grupalmente, influye notablemente en la decisión del juez, por lo que realizar de la mejor forma posible esta tarea, si bien no asegura determinada decisión, resulta ser una variable importante para una solución favorable.

El jurista al redactar los hechos de la tutela se encuentra expuesto a una complejidad superlativa que debe reducir mediante la realización de elecciones trascendentales. Debe reflexionar acerca de las posibles situaciones fácticas que pueden tener relevancia jurídica en la tutela, debe luego traducirlas a lenguaje, escoger cuidadosamente las palabras que representan dicha realidad y como ellas se conectan, y especificarlas en un documento, situación que tiene carácter argumentativo y que influencia la decisión del juez.

La acción de tutela puede asemejarse a un discurso argumentativo donde existe una contraposición de argumentos, en el que los hechos de la tutela son argumentos. En este discurso las reglas de la racionalidad judicial, pueden otorgar señales genéricas para inspirar la redacción de los hechos de la tutela. Sin embargo, de forma específica, se sugiere que la redacción de la tutela sea entendida como un proceso dinámico, complejo, reflexivo, provisional, que está conformado cuando menos por tres etapas: la preparativa, la de diseño y la de revisión.

El establecimiento de los hechos puede ser considerado bajo la óptica del test de redacción de los hechos de la tutela, en el cual se plasman doce estratagemas que implican una reflexión más consciente sobre la importancia de utilizar la palabra adecuada y el gran poder simbólico y lingüístico que esto tiene en la decisión del juez y especialmente en la suerte de quien alegue la afectación iusfundamental.

51 WRÓBLEWSKI (2003) p. 51. 


\section{BIBLIOGRAFÍA CITADA}

Alexy, Robert (2000): Teoría del discurso y derechos Humanos (Bogotá, Universidad Externado de Colombia).

AleXY, Robert (2002): Teoría de los derechos fundamentales (Madrid, Centro de Estudios Políticos y Constitucionales).

Austin, John (1990): Cómo hacer cosas con palabras: Palabras y acciones (Barcelona, Paidós).

Bernal, Carlos (2003): El principio de proporcionalidad y los derechos fundamentales (Madrid, Centro de Estudios Políticos y Constitucionales).

Bernal, Carlos (2005): "La ponderación como procedimiento para interpretar los derechos fundamentales", en CÁCeres Nieto, Enrique et al., Problemas contemporáneos de la filosofía del derecho (México, Universidad Autónoma de México).

Bernal, Carlos (2007): El Neoconstitucionalismo a debate (Bogotá, Universidad Externado). Calderón, Juan (2010): La Constitucionalización del derecho privado (Bogotá, Uniandes).

Carofiglio, Gianrico (2010): El arte de la duda (Barcelona, Marcial Pons).

Carrio, Genaro (1995): Cómo estudiar y cómo argumentar un caso (Buenos Aires, ABeledoPerrot).

DwOrKIn, Ronald (1992): El Imperio de la Justicia (Barcelona, Gedisa).

ELSTER, John (1989): Ulises y las sirenas. Estudios sobre racionalidad e irracionalidad (México, Fondo de Cultura Económica).

Foucault, Michel (1981): Nietzsche, Freud, Marx (Barcelona, Anagrama).

Frege, Gottlob (1973): Sobre el sentido y la denotación, semántica filosófica: problemas y discusiones (Madrid, Siglo XXI).

GuTIÉrREZ, Salvador (1997): La oración y sus funciones (Madrid, Arco/Libros).

Hernández, Juan (2010): Argumentación Jurídica (México, Oxford University Press).

KalinowsKi, George (1975): Lógica del discurso normativo (Madrid, Tecnos).

Lo CASCIO, Vicenzo (1998): Gramática de la argumentación. Estrategias y estructuras (Madrid, Alianza Universidad).

Lopera, Gloria y Arias, Diana (2010): Principio de proporcionalidad y derechos fundamentales en la determinación judicial de la Pena (Bogotá, Escuela Judicial Rodrigo Lara Bonilla).

Lyons, John (1997): Semántica lingüistica. Una introducción (Buenos Aires, Paidós).

Monsalve, John (2015): "La prohibición de la suspensión de los servicios públicos domiciliarios: un retorno moderado a la escuela del servicio público", Revista Iustitia, vol. V, $\mathrm{N}^{\circ}$ 12: pp. 391-415.

Nussbaum, Martha (1997): Justicia poética (Santiago de Chile, Andrés Bello).

Perelman, Chaim y Olbrechts-Tyteca, Lucie (1989): Tratado de la argumentación. La nueva retórica (Madrid Gredos).

Pizarro, Fina (1997): Aprender a Razonar (México, Editorial Alhambra Mexicana).

Robles, Gregorio (1984): Las reglas del derecho y las reglas de los juegos (México, UNAM).

Schopenhauer, Arthur (1997): Dialéctica erística o el arte de tener razón, expuesta en 38 estratagemas (Madrid, Trotta). 
SuÁrez, Wilson (2015): "El rol del juez en el Estado Constitucional", Revista Iustitia, vol. V, N ${ }^{\circ}$ 12: pp. 103-120.

Von Wright, Georg (1979): Norma y Acción (Madrid, Editorial Tecnos).

Wróblewski, Jerzy (2003): Justificación de las decisiones jurídicas, en Sentido y hecho en el derecho (México, Fontamara). 